\title{
Mitotic Kinesin Modulator
}

National Cancer Institute

\section{Source}

National Cancer Institute. Mitotic Kinesin Modulator. NCI Thesaurus. Code C67440.

A class of compounds that modulate the activity of mitotic kinesins. Mitotic kinesins or microtubule-associated proteins (MAPs) are ATP-powered proteins that convert energy into a mechanical force along the microtubule structure following the hydrolysis of ATP, which occurs during microtubule polymerization. Mitotic kinesins play an essential role in regulating the formation of the mitotic spindle as well as regulating their function. Agents that modulate the activity of kinesins, specifically those that produce inhibitory effects, interfere with mitotic spindle assembly, organization and eventually, chromosome alignment and segregation, thereby inducing mitotic arrest. 